\title{
Acute Autoimmune Hemolytic Anemia Secondary to COVID 19
}

\author{
Hamed Taheri (iD ${ }^{1}$, Roya Rafaiee (iD) ${ }^{2}$, Hamed Ghazvini (iD ${ }^{3}$, Seyedeh Masoumeh Seyedhosseini Tamijani \\ (iD) ${ }^{4}$ and Raheleh Rafaiee (iD) ${ }^{3, *}$ \\ ${ }^{1}$ Cellular and Molecular Research Center, Zahedan University of Medical Sciences, Zahedan, Iran \\ ${ }^{2}$ Department of Internal Medicine, School of Medicine, Zahedan University of Medical Sciences, Zahedan, Iran \\ ${ }^{3}$ Department of Neuroscience, School of Advanced Technologies in Medicine, Mazandaran University of Medical Sciences, Sari, Iran \\ ${ }^{4}$ Psychiatry and Behavioral Sciences Research Center, Addiction Institute, Mazandaran University of Medical Sciences, Sari, Iran \\ "Corresponding author: Department of Neuroscience, School of Advanced Technologies in Medicine, Mazandaran University of Medical Sciences, Sari, Iran. Email: \\ rachel.rafaie@yahoo.com \\ Received 2021 September 13; Revised 2021 September 18; Accepted 2021 September 21.
}

Keywords: COVID-19, Anemia, Hemolytic, Autoimmune, SARS-CoV-2

\section{Dear Editor,}

The recent severe acute respiratory syndrome coronavirus 2 (SARS-CoV-2), officially named COVID-19, is an enveloped, non-segmented positive-sense RNA virus from the beta-coronaviridae family (1). It causes severe respiratory infection as well as acute respiratory distress syndrome (ARDS), which has claimed many lives (2). Autoimmune hemolytic anemia (AIHA) causes enhanced loss of red blood cells (RBCs) mediated by anti-erythrocyte autoantibodies, which complement activation may happen (3). Its presentation ranges from no symptoms to severe consequences. Also, it may be idiopathic or due to a comorbidity. According to the currently available data, its incidence is about 1 per $100,000 /$ year (4).

To the best of our knowledge, this is the first report from Iran, showing AIHA as a rare clinical presentation of COVID-19. Here, we present a rare case of an elderly male presenting with acute AIHA who was positive for COVID-19.

We report a 68-year-old married male patient who was referred to emergency department of a hospital in Zahedan in the early phase of the COVID-19 crisis in Iran (March 24th, 2020). The patient was fully conscious (GCS: $15)$, and his complaint was the coryza, headache, dizziness, fatigue, low-grade fever, and dark urine from three days before admission. In past medical history, no medical (diabetes mellitus, hypertension, cardiopulmonary), surgical, drug, alcohol, and tobacco history has been reported. He reported no previous contact with infected cases.

Neurological, cardiac, abdominal, lung, and locomotor system examinations were normal. On admission, the blood pressure and heart rate were 90/70 mmHg and 100 beats/min, respectively, and the body temperature was $37.8^{\circ} \mathrm{C}$. Also, his respiratory rate was 16 beats/min, and his
$\mathrm{SPO}_{2}$ was $88 \%$ on room air.

Per the outside hospital report, hemoglobin was 5.1 $\mathrm{mg} / \mathrm{dL}$ (normal range, 14 - $18 \mathrm{mg} / \mathrm{dL}$ ), HCT was 17.9\% (normal range, 39 -52\%), and C-reactive protein was positive (++). The key laboratory results during his hospitalizations are summarized in Appendix 1. RT-PCR from throat swab revealed COVID-19 infection. Chest X-ray and lung CT scan were interpreted by a radiologist to indicate no classical findings of COVID -19 pneumonia on chest. His ECG on admission showed sinus tachycardia.

Regarding the aforementioned evidence, a diagnosis of AIHA secondary to COVID-19 was assumed. Twenty-four hours after the admission, the patient experienced overt blood loss per rectum, and the hemoglobin dropped 4.6. Moreover, he was baffled, and the physical exam for scleral icterus was significant. The patient was admitted to ICU, and therapy was initiated with intravenous fluids, systemic corticosteroid therapy, and remdesivir. He was transfused with six units of isogroup-packed RBCs. From the day five of hospitalization, signs of improvement in hemoglobin and hematocrit were found ( $\mathrm{Hb}: 6.4 \mathrm{mg} / \mathrm{dL}$ ). On the 8th day of admission, he was asymptomatic with a hemoglobin of $7.4 \mathrm{mg} / \mathrm{dL}$. Therefore, the patient was sent home on oral prednisolone and referred to internal medicine outpatients clinic for a weekly reevaluation. On the 2nd day after discharge, Hb level was detected 5.3 $\mathrm{mg} / \mathrm{dL}$ again. Second test of SARS-CoV-2 RNA(RT-PCR, throat swab), 10 days after the first test, showed negative results. He was admitted again to the hospital to continue his intravenous corticosteroid treatment. On the admission day, his pulmonary examination was still asymptomatic, and urine was dark. He received five units of isogroup-packed $\mathrm{RBCs}$ on the 2 nd admission, and he was discharged with $\mathrm{Hb}$ 
of $14 \mathrm{mg} / \mathrm{dL}$ on the 11 th day. He continued oral prednisolone as $12.5 \mathrm{mg}$ /daily/oral. At the time of last follow-up, the patient was alive, and he has chronically continued oral corticosteroid for more than one year.

In this study, we reported AIHA in a patient infected with COVID-19. This case is unique; first, none of the typical presentations of COVID-19 were found, and he was assumed to suffer from hemolysis with anemia, dark urine, icterus, and his test of RT-PCR for COVID-19 was positive. Second, our case did not suffer from underlying disorders such as neoplastic, lymphoproliferative, autoimmune, or infectious conditions often associated with AIHA. In line with our study, Jawed et al. reported an AIHA secondary to COVID-19 in a 50-year-old man with Chest X-ray and CT pulmonary angiogram negative for features suggestive of COVID-19 (5). Moreover, similar to our study in terms of underlying diseases, Jacobs et al. reported a 33-yearold woman with severe anemia who also had a history of hypothyroidism (Hb:1.3 g/dL; reference,12.0 - 15.0 g/dL), headache, altered mental status of SARS-CoV-2-positive test, and a chest X-ray was consistent with the infection. She had no risk factor or underlying predisposition stimulating the development of AIHA (6).

There are scanty cases of AIHA in the setting of COVID19 , the majority in the previously immune dysregulated patients (lymphoid neoplasia and autoimmune diseases) or diabetes mellitus and hypertension. For instance, Lazarian et al. reported seven cases with COVID-19 who also had signs of hemolysis after the admission. They reported that all cases showed risk factors for severe COVID-19 such as increased blood pressure, diabetes, and chronic renal failure that all required treatment with either steroids or transfusion (7). Moreover, Brito et al. reported a patient with acute and severe AIHA after receiving the second dose of the COVID-19 messenger ribonucleic acid (mRNA) vaccine (8).

The exact etiology of autoimmune diseases is still unknown, but there are various factors, including viral infections, that contribute to triggering these type of diseases. As acquired hemolysis, the AIHA is caused by an immune response that results in the destruction of red cell antigens. In such cases, the sign of anemia may be presented, including dizziness, fatigue, and dyspnea, or evidence of hemolysis with jaundice and dark urine. Several causes are reported for AIHA, including autoimmune, viral, lymphoproliferative diseases, and immunodeficiency states. Common laboratory presentations of AIHA include anemia that may not be found in those who suffer from mild hemolysis. The white blood cells and platelets are often normal, while leukopenia or leukocytosis may be observed because of viral infection or a bone marrow disease. On a blood film, red blood cell agglutination and spherocytosis may be apparent. In most cases, the number of reticulocytes increases; however, it can be normal for a very short duration of hemolysis or with an underlying bone marrow disorder. Meanwhile, LDH and bilirubin may increase, while haptoglobin may decline.

Blood group of our patient is $\mathrm{A}+(\mathrm{RH}$, positive). Some previous studies reported that $\mathrm{ABO}$ blood groups had various associations with SARS-CoV-2 infection, and reported that blood groups of $\mathrm{A}$ and $\mathrm{O}$ were associated with enhanced and decreased risk of infection, respectively $(9,10)$.

In conclusion, this is the first reported case of AIHA in a patient infected with COVID-19 from Iran. Few reports of patients with AIHA and COVID-19 are published; nevertheless, most cases in these reports also had other comorbidities proved to be associated with autoantibodies and AIHA. This case demonstrates the fact that SARS-CoV-2, the causative agent of COVID-19, may itself be capable of inducing severe AIHA even in cases with no underlying predisposition. Given the important inflammatory state caused by the COVID-19, AIHA should be considered an immunologic complication associated with COVID-19.

\section{Supplementary Material}

Supplementary material(s) is available here [To read supplementary materials, please refer to the journal website and open PDF/HTML].

\section{Footnotes}

Authors' Contribution: All authors had equal roles in performing this research and contributed to writing the paper.

Conflict of Interests: The authors declare that they have no conflict of interest.

Ethical Approval: The Ethics Committee of Mazandaran University of Medical Sciences approved this study (ethical registration code: IR.MAZUMS.REC.1400.317).

Funding/Support: This study was supported in part by grant 10501 from the Mazandaran University of Medical Sciences.

Informed Consent: Written informed consent was obtained from the patient for publication of this case report.

\section{References}

1. Sreh AA, Jameel I, Musleh H, Shankaran V, Meghjee SP. COVID 19 and adenovirus multi-lobar pneumonia on CT scan in a patient with repeatedly normal chest X-rays despite severe hypoxia and the need for non-invasive ventilation. Cureus. 2021;13(1). e12955. 
doi: 10.7759/cureus.12955. [PubMed: 33654626]. [PubMed Central: PMC7916640].

2. Wu C, Chen X, Cai Y, Xia J, Zhou X, Xu S, et al. Risk factors associated with acute respiratory distress syndrome and death in patients with Coronavirus disease 2019 pneumonia in Wuhan, China. JAMA Intern Med. 2020;180(7):934-43. doi: 10.1001/jamainternmed.2020.0994. [PubMed: 32167524]. [PubMed Central: PMC7070509].

3. Barcellini W, Giannotta J, Fattizzo B. Autoimmune hemolytic anemia in adults: Primary risk factors and diagnostic procedures. Expert Rev Hematol. 2020;13(6):585-97. doi: 10.1080/17474086.2020.1754791. [PubMed: 32274943].

4. Hill QA, Stamps R, Massey E, Grainger JD, Provan D, Hill A, et al. The diagnosis and management of primary autoimmune haemolytic anaemia. Br J Haematol. 2017;176(3):395-411. doi: 10.1111/bjh.14478. [PubMed: 28005293].

5. Jawed M, Hart E, Saeed M. Haemolytic anaemia: A consequence of COVID-19. BMJ Case Rep. 2020;13(12). doi: 10.1136/bcr-2020-238118. [PubMed: 33303503]. [PubMed Central: PMC7733228].

6. Jacobs J, Eichbaum Q. COVID-19 associated with severe autoimmune hemolytic anemia. Transfusion.2021;61(2):635-40. doi: 10.1111/trf.16226.
[PubMed: 33274459]. [PubMed Central: PMC7753740].

7. Lazarian G, Quinquenel A, Bellal M, Siavellis J, Jacquy C, Re D, et al. Autoimmune haemolytic anaemia associated with COVID-19 infection. Br J Haematol. 2020;190(1):29-31. doi: 10.1111/bjh.16794. [PubMed: 32374906]. [PubMed Central: PMC7267601].

8. Brito S, Ferreira N, Mateus S, Bernardo M, Pinto B, Lourenco A, et al. A case of autoimmune hemolytic anemia following COVID-19 messenger ribonucleic acid vaccination. Cureus. 2021;13(5). e15035. doi: 10.7759/cureus.15035. [PubMed: 34150386]. [PubMed Central: PMC8200779].

9. Zhao J, Yang Y, Huang H, Li D, Gu D, Lu X, et al. Relationship between the $\mathrm{ABO}$ blood group and the Coronavirus disease 2019 (COVID-19) susceptibility. Clin Infect Dis. 2021;73(2):328-31. doi: 10.1093/cid/ciaa1150. [PubMed: 32750119]. [PubMed Central: PMC7454371].

10. Fan Q, Zhang W, Li B, Li DJ, Zhang J, Zhao F. Association between ABO blood group system and COVID-19 susceptibility in Wuhan. Front Cell Infect Microbiol. 2020;10:404. doi: 10.3389/fcimb.2020.00404. [PubMed: 32793517]. [PubMed Central: PMC7385064]. 